\title{
Comparison of diagnostic accuracy of screening tests ALT and ultrasound for pediatric non-alcoholic fatty liver disease
}

\author{
Laura G. Draijer ${ }^{1}$ (D) Sana Feddouli ${ }^{1}$ - Anneloes E. Bohte ${ }^{2}$. Olga vd Baan Slootweg ${ }^{3} \cdot$ Tammo H. Pels Rijcken $^{4}$.
} Marc A. Benninga ${ }^{1} \cdot$ Jaap Stoker ${ }^{2} \cdot$ Bart G. P. Koot ${ }^{1}$

Received: 12 January 2019 / Revised: 3 March 2019 / Accepted: 4 March 2019 / Published online: 22 March 2019

(C) The Author(s) 2019

\begin{abstract}
Alanine aminotransferase (ALT) and ultrasound (US) are the most commonly used tools for detecting non-alcoholic fatty liver disease (NAFLD). No direct comparison of these two modalities in children exists. We aimed to compare head-to-head the diagnostic accuracy of ALT and US and their combination for detecting NAFLD in children with obesity. Ninety-nine children with severe obesity underwent simultaneous serum-ALT and abdominal ultrasound (US steatosis score 0-3). Proton magnetic resonance spectroscopy was used as reference standard for detecting steatosis/NAFLD. ROC curve analyses were performed to determine diagnostic performance and to determine optimum screening cut-points aiming for a specificity $\geq 80 \%$. The area under the ROC (AUROC) of ALT and US were not significantly different (0.74 and 0.70, respectively). At the optimal ALT threshold ( $\geq 40 \mathrm{IU} / \mathrm{L}$ ), sensitivity was $44 \%$ and specificity was $89 \%$. At the optimal US steatosis score $(\geq 2)$, sensitivity was $51 \%$ and specificity was $80 \%$. Combining ALT and US did not result in better accuracy than ALT or US alone.

Conclusion: ALT and US have comparable and only moderate diagnostic accuracy for detecting hepatic steatosis in children with obesity. A stepwise screening strategy combining both methods does not improve diagnostic accuracy.

What is Known:

- Alanine aminotransferase (ALT) and ultrasound (US) are the most commonly used tools for detecting non-alcoholic fatty liver disease (NAFLD).

- ALT and ultrasound have mediocre accuracy in detecting steatosis in children with obesity.

- Comparing accuracy data from previous studies on the accuracy of these screening tests is difficult as study populations and reference standards differ among studies.
\end{abstract}

What is New:

- In a head-to-head comparison, the difference in diagnostic accuracy of ALT and ultrasound in detecting steatosis is not significant.

- A stepwise screening strategy combining both methods does not improve diagnostic accuracy.

Communicated by Peter de Winter

Laura G. Draijer

1.g.draijer@amc.uva.nl

Sana Feddouli

s.feddouli@hotmail.com

Anneloes E. Bohte

a.e.bohte@amc.uva.nl

Olga vd Baan Slootweg

baan0337@ planet.nl

Tammo H. Pels Rijcken

tammopr@gmail.com

Marc A. Benninga

m.a.benninga@amc.uva.nl

Jaap Stoker

j.stoker@amc.uva.nl
Bart G. P. Koot

b.g.koot@amc.uva.nl

1 Department of Pediatric Gastroenterology and Nutrition, Amsterdam University Medical Centers, Location Academic Medical Center/ Emma Children's Hospital, Meibergdreef 9, 1100 AZ Amsterdam, The Netherlands

2 Department of Radiology and Nuclear Medicine, Amsterdam University Medical Centers, Location Academic Medical Center, Amsterdam, The Netherlands

3 Obesity Clinic Heideheuvel, Soestdijkerstraatweg 129, 1213 VX Hilversum, The Netherlands

4 Department of Radiology, Tergooi Hospital, Van Riebeeckweg 212, 1213 XZ Hilversum, The Netherlands 
Keywords NAFLD $\cdot$ Children $\cdot$ Obesity $\cdot$ Screening $\cdot$ ALT $\cdot$ Ultrasound

\begin{tabular}{ll}
\multicolumn{2}{l}{ Abbreviations } \\
ALT & Alanine aminotransferase \\
AST & Aspartate aminotransferase \\
AUROC & Area under the ROC curve \\
BMI & Body mass index \\
${ }^{1}$ H-MRS & Proton magnetic resonance spectroscopy \\
H O M A & -Homeostatic model assessment of insulin resis- \\
IR & tance score \\
IQR & Interquartile range \\
LR+ & Positive likelihood ratio \\
LR- & Negative likelihood ratio \\
NAFLD & Non-alcoholic fatty liver disease \\
NPV & Negative predictive value \\
PPV & Positive predictive value \\
ROC & Receiver operating characteristic \\
SD & Standard deviation \\
STARD & Standards for reporting of diagnostic accuracy \\
ULN & Upper limit of normal \\
US & Ultrasound \\
US score & Ultrasound steatosis score \\
$\gamma$ GT & Gamma-glutamyltransferase
\end{tabular}

\section{Introduction}

Non-alcoholic fatty liver disease (NAFLD) is well established as one of the complications of obesity. NAFLD includes different stages of disease: simple steatosis, steatohepatitis, fibrosis, and cirrhosis. The reported prevalence of NAFLD in children is $7.6 \%$ in general population studies and $38 \%$ in studies based on child obesity clinics [1-3]. Concomitant with the rise of obesity, the prevalence of NAFLD has increased during the last two decades, making NAFLD the most common chronic liver disease in children in the industrialized world $[2,4]$.

It is important to identify patients with obesity and NAFLD since advanced fibrosis is reported in up to $17 \%$ of children referred to liver centers after screening $[4,5]$. In view of their long life expectancy, patients with significant fibrosis at pediatric age are particularly at risk of long-term complications during their lifetime, i.e., cirrhosis, liver failure, and hepatocellular carcinoma. In addition, NAFLD is also an independent risk factor for type 2 diabetes and, although still disputed, probably also for cardiovascular disease at adult age $[6,7]$.

Because of these long-term health risks and lack of symptoms until advanced disease occurs, screening for NAFLD in children with obesity is propagated in most major national and international obesity and hepatology guidelines [8]. The first step in screening is usually to detect steatosis, and subsequently, those patients identified are further evaluated for inflammation and fibrosis. Guidelines differ in their advice on the primary screening tool, alanine aminotransferase (ALT), and ultrasound (US). This disparity is due to the lack of data on the optimal primary screening tool to detect NAFLD.

Although the use of ALT and US as screening tools have been evaluated in previous studies, no study compares headto-head the diagnostic accuracy of ALT and US [9-15]. Also, a stepwise screening approach that combines both tests has not yet been studied. The aim of the present study was to compare head-to-head the accuracy of ALT and US as screening tools for NAFLD in children with severe obesity. In addition, we evaluated whether a stepwise screening approach combining both tests increases the accuracy of screening.

\section{Methods}

\section{Study design and patients}

This was a cross-sectional study. Participants were recruited from the obesity clinic "Heideheuvel" in Hilversum, the Netherlands, during a 2-year study period between February 2008 and October 2010. Admission criteria for this program were severe obesity (body mass index $z$-score $>2.6$ ) or obesity (BMI $z$-score $>2$ ) along with obesity-related co-morbidity (dyslipidemia and hypertension). All children admitted to the obesity clinic between 8 and 18 years old were eligible for participation in our study. Exclusion criteria were known liver disease other than NAFLD (viral/autoimmune hepatitis, Wilson's disease, hemochromatosis, alpha-1 antitrypsin deficiency), metabolic disease (b-oxidation defects, urea cycle defects), (history of) use of steatogenic medication, alcohol consumption $>7$ units/ week, jejunoileal surgery, type 1 or type 2 diabetes mellitus, history of parenteral feeding, and contraindications for MRI. All participants underwent an ultrasound of the liver, venipuncture and a proton magnetic resonance spectroscopy $\left({ }^{1} \mathrm{H}\right.$-MRS) of the liver before starting the lifestyle program at the obesity clinic within a time interval of 1 month. Accuracy data on ultrasound of a part of this study population were previously published by Bohte et al. [16]. The study was approved by the Medical Ethics Committee of the Academic Medical Center of the University of Amsterdam. Written informed consent was obtained from the participants and/or their legal guardians. We followed the Standards for Reporting of Diagnostic Accuracy (STARD) guidelines in this study on the accuracy of ALT and US in detecting hepatic steatosis [17]. 


\section{Clinical assessment}

All measurements were performed at the start of the lifestyle program at the obesity clinic. Weight and length were measured to calculate the BMI $z$-score. The BMI $z$-score is the number of standard deviations (SD) from the mean on a standard BMI curve for age and gender. Children with a BMI zscore of $>2$ (95th percentile) are considered obese, and those with a BMI $z$-score of $>2.6$ (99th percentile) are considered severely obese $[18,19]$.

\section{Laboratory tests}

Venous blood was sampled after an overnight fast for serum biochemistry studies. ALT, aspartate aminotransferase (AST), gamma-glutamyltransferase $(\gamma \mathrm{GT})$ and lipids were measured directly after blood sampling using standard laboratory methods by certified laboratory staff. Fasted insulin and glucose were used to calculate the homeostatic model assessment of insulin resistance score (HOMA-IR) [20]. An oral glucose tolerance test was performed to exclude the presence of type 2 diabetes. Hepatitis B and C, autoimmune hepatitis, alpha-1 antitrypsin deficiency, abetalipoproteinemia, hemochromatosis, and Wilson's disease were excluded using the appropriate diagnostic tests.

\section{Index test 1: Alanine transaminase}

We determined the accuracy of ALT at cutoffs commonly applied in screening guidelines: the sex-specific upper limit of normal (ULN) for healthy children (22 IU/L for girls and $26 \mathrm{IU} / \mathrm{L}$ for boys) [21]; $2 \times$ the sex-specific ULN (44 IU/L for girls and 52 IU/L for boys) [22]; and the cutoff mostly used by laboratories and physicians in clinical practice for both sexes (ALT $\geq 40 \mathrm{IU} / \mathrm{L})$.

\section{Index test 2: Ultrasound}

For this study, we used US systems (ATL HDI 5000, HD11, and IU22; Philips Healthcare, Best, the Netherlands) with 25- and 3.75-MHz curved-array transducers. The "abdominal general" setting was used on the US system for all the US examinations. Three radiologists with 5-20 years' experience and more than 600 liver US examinations per year, performed and interpreted the US examination of all participants. All three radiologists were blinded for the data of this study. The following acknowledged scoring items were used: (1) echogenicity of liver parenchyma; (2) visualization of the diaphragm; (3) visualization of intrahepatic vessels; and (4) visualization of posterior part of the right hepatic lobe. Subsequently, the degree of liver steatosis was scored (Table 1). The "ultrasound steatosis score" (US score) was defined as the average score of the four items [23]. We determined the accuracy of US using each US score. As previously published, the interobserver agreement between the three radiologists was moderate to good (kappa 0.58 to 0.68 ) and the intraobserver agreement (kappa 0.82 to 0.91 ) was excellent.

\section{Reference test: Proton magnetic resonance spectroscopy}

${ }^{1} \mathrm{H}-\mathrm{MRS}$ spectra were acquired using a point-resolved spectroscopy sequence (TE/TR $=38 / 2000 \mathrm{~ms}$ ) in a voxel of $20 \times$ $20 \times 20 \mathrm{~mm}^{3}$ during free breathing on a 3.0 Tesla MR system (Philips Healthcare, Best, the Netherlands). An open bore 1.0 Tesla MR scanner (Philips Healthcare, Best, the Netherlands) was used in participants with body weight more than $150 \mathrm{~kg}$ or an abdominal circumference of more than $150 \mathrm{~cm}$. In this study, the presence of steatosis is defined as a liver fat percentage of $>1.8 \%$ measured by ${ }^{1} \mathrm{H}-\mathrm{MRS}$. This cutoff has been validated to correspond with $>5 \%$ fat containing hepatocytes on liver histology, i.e., the histological definition of NAFLD [24]. ${ }^{1} \mathrm{H}-\mathrm{MRS}$ spectra were analyzed by a research fellow with 3 years of experience and supervised by an MR physicist with 8 years of experience. Both were blinded for the results of ALT and US. ${ }^{1} \mathrm{H}-\mathrm{MRS}$ is an accurate non-invasive tool to diagnose or exclude NAFLD [25].

\section{Statistical analysis}

Patients' demographic data, laboratory and imaging data were summarized with mean and standard deviation for continuous
Table 1 Scoring of hepatic steatosis with ultrasound (US score)

\begin{tabular}{ll}
\hline Score $0=$ normal & $\begin{array}{c}\text { Normal echogenicity of liver parenchyma. Normal visualization of the diaphragm and } \\
\text { intrahepatic blood vessels. }\end{array}$ \\
$\begin{array}{c}\text { Score } 1=\text { mild } \\
\text { steatosis }\end{array}$ & $\begin{array}{c}\text { Slightly increased echogenicity of liver parenchyma. Normal visualization of the } \\
\text { diaphragm and intrahepatic blood vessels. }\end{array}$ \\
Score $2=$ moderate & Markedly increased echogenicity of liver parenchyma. Slightly decreased visualization \\
steatosis & of the diaphragm and intrahepatic blood vessels. \\
Score $3=$ severe & Severely increased echogenicity of liver parenchyma. No or severely decreased \\
steatosis & visualization of the diaphragm, intrahepatic blood vessels, and posterior part of the \\
& right liver lobe.
\end{tabular}

US score ultrasound steatosis score 
variables with a normal distribution or median and interquartile range (IQR) for continuous variables with a non-normal distribution. Receiver operating characteristic (ROC) analysis was used to evaluate the discriminatory power of the two screening tests. We compared the areas under the ROC curves (AUROC) in MedCalc by using a (pairwise) comparison according to the method used by Hanley and McNeil [26]. A value of $p<0.05$ was considered a statistically significant difference. In addition, at the aforementioned ALT cutoffs and for all US scores, the diagnostic accuracy was calculated, including sensitivity, specificity, positive predictive value (PPV), negative predictive value (NPV), and positive and negative likelihood ratios $(\mathrm{LR}+, \mathrm{LR}-)$ with $95 \%$ confidence intervals.

Subsequently, ALT and US were combined in a stepwise screening approach using the previously calculated optimal cutoffs of both tools. Diagnostic accuracy of those algorithms was calculated, including total accuracy in order to assess overall performance. The total accuracy is defined as (prevalence $\times$ sensitivity $) /((1-$ prevalence $) \times$ specificity $)[27]$.

Since NAFLD has a high prevalence among children with obesity and missing the diagnosis has no direct harmful health effect, it is rational to primarily aim for an adequate specificity to limit the number of false positives and thereby avoid unnecessary distress among patients and a huge burden of unnecessary additional testing. In this study, we defined an adequate specificity as $>80 \%$.

All analyses were performed with PASW Statistics 18 SPSS Inc., Chicago, IL; MedCalc Software; and with Microsoft Office Excel, Microsoft, Redmond, WA.

\section{Results}

\section{Participants}

In this study, 121 participants were consecutively included. Two participants withdrew from the study and the data on US and ${ }^{1} \mathrm{H}-\mathrm{MRS}$ were incomplete in 20 participants. In total, data of 99 children ( 42 male, 57 female) were analyzed. The mean age was $14.1 \pm 2.1$ years (range $8.3-17.8$ ). The mean BMI $z$-score was $3.3 \pm 0.3 \mathrm{SD}$ (range 2.5-4.2). The baseline characteristics are summarized in Table 2. Hepatic steatosis was present in 43/99 children (43.4\%).

\section{Diagnostic accuracy}

The ROC curves of ALT and US for detection of hepatic steatosis are presented in Fig. 1. The AUROC values of ALT and US were comparable: 0.74 (95\% CI $0.65-0.83$ ) and 0.70 (95\% CI $0.60-0.79)$, respectively $(p=0.41)$.
Table 2 Baseline characteristics

\begin{tabular}{ll}
\hline Demographic & \\
Age (years) & $14.1(2.1)$ \\
Female, $n(\%)$ & $57(57.6)$ \\
Clinical & \\
Steatosis, $n(\%)$ & $43(43.4)$ \\
BMI $z$-score & $3.3(0.3)$ \\
Waist circumference, cm (IQR) & $102(14)$ \\
Biological data & \\
ALT, IU/L (IQR) & $27(22)$ \\
$\gamma$ GT, IU/L (IQR) & $21(9)$ \\
HOMA-IR (IQR) & $3.17(2.5)$ \\
Triglycerides, mmol/L (IQR) & $0.85(0.60)$ \\
Total cholesterol, mmol/L & $4.02(0.79)$ \\
HDL-cholesterol, mmol/L & $1.07(0.23)$ \\
LDL-cholesterol, mmol/L & $2.52(0.69)$ \\
Imaging data & \\
US steatosis score $0, n(\%)$ & $23(23.2)$ \\
US steatosis score $1, n(\%)$ & $42(42.4)$ \\
US steatosis score $2, n(\%)$ & $25(25.3)$ \\
US steatosis score 3, $n(\%)$ & $9(9.1)$ \\
\hline
\end{tabular}

Continuous variables are expressed in mean with standard deviation in parentheses or median with interquartile range (IQR) in parentheses or $n$ (\%). BMI body mass index, $A L T$ alanine aminotransferase, $A S T$ aspartate aminotransferase, $\gamma G T$ gamma-glutamyltransferase, HOMA-IR homeostatic model assessment of insulin resistance, $H D L$ high-density lipoprotein, $L D L$ low-density lipoprotein, $U S$ ultrasound

\section{ALT}

The diagnostic accuracy of ALT at the aforementioned cutoffs is presented in Table 3. At a threshold of ALT $\geq 40 \mathrm{IU} / \mathrm{L}$, the predetermined minimal specificity of $80 \%$ was reached, however, with a sensitivity of only $44 \%$.

\section{Ultrasound}

The diagnostic accuracy of US at different US scores is presented in Table 4. A US score $\geq 2$ reached the predetermined minimal specificity of $>80 \%$, however, with a sensitivity of only $51 \%$.

\section{Stepwise screening strategy}

Different stepwise approaches combining ALT and US were evaluated. In the first algorithm, all patients with ALT $\geq 40 \mathrm{IU} /$ L undergo additional US to increase the specificity. Only those with $\mathrm{ALT} \geq 40 \mathrm{IU} / \mathrm{L}$ plus US score $\geq 2$ were considered to have a positive screening result. In the second algorithm, to increase the sensitivity, US is performed in all patients with ALT $<40 \mathrm{IU} / \mathrm{L}$. Patients with ALT $\geq 40 \mathrm{IU} / \mathrm{L}$ and those with ALT $<40 /$ IU/L plus US score $\geq 2$ were considered to have a 
Fig. 1 ROC curves of ALT and US. The AUROC of ALT and US were 0.70 (95\% CI $0.60-0.79$ ) and 0.74 (95\% CI 0.65-0.83), respectively (NS). ROC, receiver operating curve; ALT, alanine aminotransferase; US, ultrasound; AUROC, area under the ROC curve

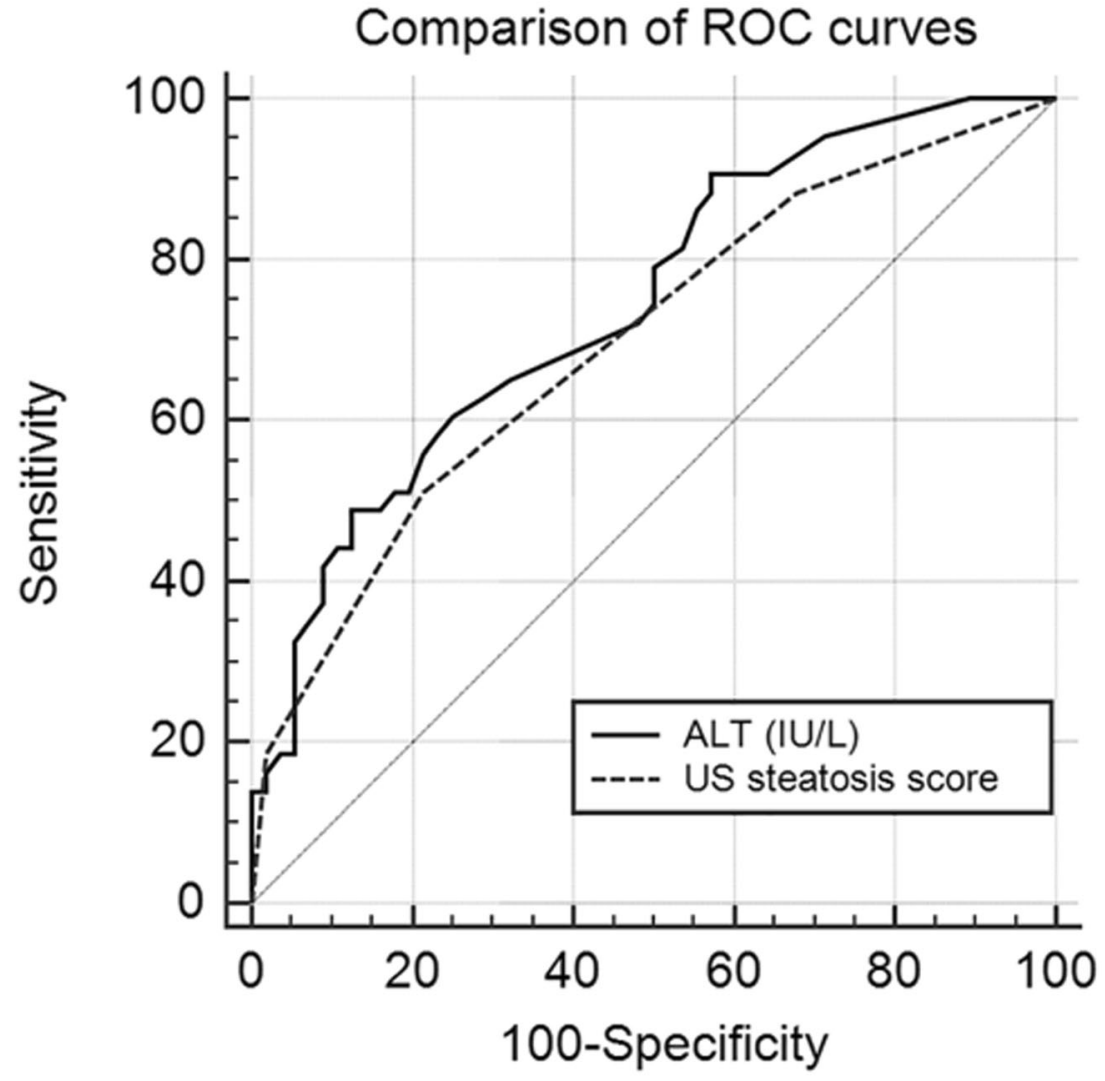

positive screening result in this algorithm. Thirdly, to limit the number of additional ultrasounds, we determined if performing ultrasound in the group of patients with ALT $\geq$ ULN but $<40 \mathrm{IU} / \mathrm{L}$ increases the accuracy. Patients with ALT levels $\geq 40 \mathrm{IU} / \mathrm{L}$ and those with $\mathrm{ALT} \geq \mathrm{ULN}$ but $<40 \mathrm{IU} / \mathrm{L}$ plus US score $\geq 2$ were considered to have a positive screening result. In the last algorithm, to increase the specificity, ultrasound is performed in all patients with ALT $>$ ULN (Table 5). A combination of ALT $\geq 40$ IU/L and US score $\geq$ 2 increases the specificity of screening to $98 \%$ but decreases the sensitivity to $32 \%$. The overall accuracy of this algorithm was $70 \%$, which is comparable to the overall accuracy of ALT $\geq 40 \mathrm{IU} / \mathrm{L}$ alone (overall accuracy of $70 \%$ ). All other algorithms also had a comparable and only mediocre overall accuracy. We also performed additional analyses assessing the algorithms using US as a first test, followed by ALT measurement. These combinations did not improve the accuracy of screening (data not shown).

\section{Discussion}

To our knowledge, this is the first study that compares head-to-head the accuracy of ALT and US as screening tools for NAFLD in children with obesity. We show that none of the currently used screening tools for
Table 3 Diagnostic accuracy of alanine aminotransferase (ALT)

\begin{tabular}{lllllll}
\hline $\begin{array}{l}\text { Test } \\
(\mathrm{IU} / \mathrm{L})\end{array}$ & $\begin{array}{l}\text { Sensitivity } \% \\
(95 \% \mathrm{CI})\end{array}$ & $\begin{array}{l}\text { Specificity } \% \\
(95 \% \mathrm{CI})\end{array}$ & $\begin{array}{l}\text { PPV \% } \\
(95 \% \mathrm{CI})\end{array}$ & $\begin{array}{l}\text { NPV \% } \\
(95 \% \mathrm{CI})\end{array}$ & $\begin{array}{l}\text { LR+ } \\
(95 \% \mathrm{CI})\end{array}$ & $\begin{array}{l}\text { LR- } \\
(95 \% \mathrm{CI})\end{array}$ \\
\hline ALT $\geq$ ULN & 79 & 50 & 55 & 76 & 1.6 & 0.4 \\
$n=62$ & $(64-90)$ & $(36-64)$ & $(47-62)$ & $(62-85)$ & $(1.2-2.1)$ & $(0.2-0.8)$ \\
$\mathrm{ALT} \geq 40$ & 44 & 89 & 76 & 68 & 4.1 & 0.6 \\
$n=25$ & $(29-60)$ & $(78-96)$ & $(58-88)$ & $(61-73)$ & $(1.8-9.4)$ & $(0.5-0.8)$ \\
$\mathrm{ALT} \geq 2 \times$ ULN & 23 & 95 & 77 & 62 & 4.3 & 0.8 \\
$n=13$ & $(12-39)$ & $(85-99)$ & $(49-92)$ & $(57-66)$ & $(1.3-14.8)$ & $(0.7-1.0)$ \\
\hline
\end{tabular}

$\mathrm{ULN}=22 \mathrm{IU} / \mathrm{L}$ for girls, $26 \mathrm{IU} / \mathrm{L}$ for boys

$A L T$ alanine aminotransferase, $U L N$ upper limit of normal, $P P V$ positive predictive value, $N P V$ negative predictive value, $L R+$ positive likelihood ratio, $L R-$ negative likelihood ratio 
Table 4 Diagnostic accuracy of ultrasound (US)

\begin{tabular}{lllllll}
\hline Test & $\begin{array}{l}\text { Sensitivity } \% \\
(95 \% \mathrm{CI})\end{array}$ & $\begin{array}{l}\text { Specificity } \% \\
(95 \% \mathrm{CI})\end{array}$ & $\begin{array}{l}\text { PPV \% } \\
(95 \% \mathrm{CI})\end{array}$ & $\begin{array}{l}\text { NPV \% } \\
(95 \% \mathrm{CI})\end{array}$ & $\begin{array}{l}\text { LR+ } \\
(95 \% \mathrm{CI})\end{array}$ & $\begin{array}{l}\text { LR- } \\
(95 \% \mathrm{CI})\end{array}$ \\
\hline $\mathrm{US} \geq 1$ & 88 & 32 & 50 & 78 & 1.3 & 0.4 \\
$n=76$ & $(75-96)$ & $(20-46)$ & $(44-55)$ & $(59-90)$ & $(1.1-1.6)$ & $(0.2-0.9)$ \\
$\mathrm{US} \geq 2$ & 51 & 80 & 65 & 68 & 2.4 & 0.6 \\
$n=34$ & $(35-67)$ & $(66-88)$ & $(51-77)$ & $(60-75)$ & $(1.3-4.3)$ & $(0.4-0.9)$ \\
$\mathrm{US} \geq 3$ & 19 & 98 & 89 & 61 & 10.4 & 0.8 \\
$n=9$ & $(8-33)$ & $(90-100)$ & $(51-98)$ & $(58-64)$ & $(1.4-80.1)$ & $(0.7-1.0)$ \\
\hline
\end{tabular}

US ultrasound steatosis score, $P P V$ positive predictive value, $N P V$ negative predictive value, $L R+$ positive likelihood ratio, $L R-$ negative likelihood ratio
NAFLD is superior. Both ALT and US have only a mediocre accuracy (AUROC 0.74 (95\% CI 0.65-0.83) and 0.70 (95\% CI 0.60-0.79), respectively). When using these screening tools in practice, we consider a threshold of ALT $\geq 40$ IU/L or US score $\geq 2$ to be optimal since at these thresholds the specificity is acceptable $(\geq 80 \%)$, albeit combined with a low sensitivity (44\% and $51 \%$, respectively). In practice, for reasons of costs and availability, we consider ALT the preferred screening tool in most settings.

Ideally, a screening test has a high specificity of $>95 \%$ (i.e., $<5 \%$ false positives) and a positive likelihood ratio of $>10$ [28]. Our results show that for both ALT and US as screening tools for NAFLD, these criteria cannot be met unless the cutoff is put very high, resulting in an unacceptably low sensitivity. For example, using a US score $\geq 3$, a specificity of $98 \%$ and a LR+ of 18 can be obtained. However, when using this cutoff, sensitivity is only $19 \%$. In other words, $81 \%$ of the children with NAFLD will not be identified.

The results from this study underscore that physicians applying these diagnostic tests should be aware of the limited accuracy of the tests, particularly realizing that a negative screening result does not exclude NAFLD. Albeit not in the scope of this study, physicians should also be aware that ALT and US correlate not/very poorly with the presence of inflammation and fibrosis [29-31]. Thus, these tests do not provide information on the stage of NAFLD. It is important that patients are counseled on these aspects when discussing screening results.

Although no head-to-head studies comparing ALT and US exist, several pediatric studies evaluated ALT and US as screening tools for NAFLD. Comparing accuracy data from these studies is difficult as study populations and reference standards differ among studies. Two previous studies on the accuracy of US for detecting NAFLD in children with obesity using MRI as reference standard reported higher sensitivity (95\% and $93 \%$ ) and specificity (50\% and $70 \%$ ) compared with our study $[9,10]$. The study population of these studies was less severely obese compared with that of our study (mean BMI $z$-score 2.5 in Pacifico et al. and 2.2 in Pozatto et al.). In addition, in these studies, a threshold of 9\% MRI determined liver fat was used, without validation. This threshold is probably compatible with the detection of moderate to severe steatosis, rather than all degrees of steatosis. Likewise, in a study in children treated in a tertiary liver clinic, thus not on a population level, a US score $\geq 2$ was found to have a good

Table 5 Diagnostic accuracy characteristics of stepwise screening strategy

\begin{tabular}{llllllll}
\hline Test & $\begin{array}{l}\text { Sensitivity } \% \\
(95 \% \mathrm{CI})\end{array}$ & $\begin{array}{l}\text { Specificity } \% \\
(95 \% \mathrm{CI})\end{array}$ & $\begin{array}{l}\text { PPV \% } \\
(95 \% \mathrm{CI})\end{array}$ & $\begin{array}{l}\text { NPV \% } \\
(95 \% \mathrm{CI})\end{array}$ & $\begin{array}{l}\text { LR+ } \\
(95 \% \mathrm{CI})\end{array}$ & $\begin{array}{l}\text { LR- } \\
(95 \% \mathrm{CI})\end{array}$ & $\begin{array}{l}\text { Overall accuracy \% } \\
(95 \% \mathrm{CI})\end{array}$ \\
\hline $\mathrm{ALT} \geq 40$ and US $\geq 2$ & 33 & 98 & 93 & 65 & 18.0 & 0.7 & 70 \\
$n=15$ & $(19-49)$ & $(90-100)$ & $(66-99)$ & $(61-70)$ & $(2.5-133.3)$ & $(0.6-0.9)$ & $(60-79)$ \\
$\mathrm{ALT}<40$ and US $\geq 2$ & 63 & 70 & 61 & 71 & 2.1 & 0.5 & 67 \\
$n=44$ & $(47-77)$ & $(56-81)$ & $(50-72)$ & $(61-79)$ & $(1.3-3.3)$ & $(0.4-0.8)$ & $(56-76)$ \\
$\mathrm{ULN} \leq \mathrm{ALT}<40$ and US $\geq 2$ & 56 & 71 & 60 & 68 & 2.0 & 0.6 & 65 \\
$n=40$ & $(40-71)$ & $(78-83)$ & $(48-71)$ & $(59-75)$ & $(1.2-3.2)$ & $(0.4-0.9)$ & $(54-74)$ \\
$\mathrm{ALT} \geq \mathrm{ULN}$ and US $\geq 2$ & 44 & 80 & 63 & 65 & 2.3 & 0.7 & 65 \\
$n=30$ & $(29-60)$ & $(68-90)$ & $(48-76)$ & $(58-72)$ & $(1.2-4.2)$ & $(0.5-0.9)$ & $(54-74)$ \\
\hline
\end{tabular}

$\mathrm{ULN}=22 \mathrm{IU} / \mathrm{L}$ for girls, $26 \mathrm{IU} / \mathrm{L}$ for boys

$U L N$ upper limit of normal, $U S$ ultrasound, $A L T$ alanine aminotransferase, $P P V$ positive predictive value, $N P V$ negative predictive value, $L R+$ positive likelihood ratio, $L R-$ negative likelihood ratio 
accuracy (sensitivity $80 \%$, specificity $86 \%$ ) for detecting moderate to severe steatosis using histology as the reference standard [11]. As previously published, in our study cohort, the accuracy of US for detecting moderate to severe steatosis is indeed higher than that for detecting all stages of steatosis [16]. Detecting mild steatosis is however equally important as detecting more severe degrees of steatosis as there is no association between the intensity of steatosis and fibrosis grade [32]. Previous studies on ALT as a screening tool in children with obesity reported a slightly better but still mediocre accuracy for detecting steatosis [9, 12-15]. Again, in these studies, children had less severe obesity compared with our study population [13] or included patients with normal BMI as well [14, $15]$.

As a secondary aim of this study, we evaluated whether a stepwise screening approach combining both tests would increase the accuracy of screening. None of the algorithms had an overall accuracy superior to ALT $\geq 40 \mathrm{IU} / \mathrm{L}$ alone. Thus, a stepwise screening strategy using ALT and US consecutively does not improve the accuracy of screening. This implies that combining both tools is not a useful strategy in the screening for NAFLD in children with obesity.

The strength of this study is that it was performed in a cohort of patients that were not selected on liver abnormalities and performed in an obesity clinic. Previous studies reporting on the accuracy of screening tools for pediatric NAFLD were frequently conducted in patients referred to hepatology departments of tertiary care hospitals, resulting in highly selected cohorts often of patients that required liver biopsy based on clinical or biochemical abnormalities. Also, all participants were well characterized and underwent a standardized liver disease assessment to exclude other causes of liver disease before inclusion. A limitation of this study is that the study population had severe obesity (mean BMI $z$-score 3.3 ) which might not be representative of the population in all obesity clinics. Performing a head-to-head comparison in children with less severe obesity is valuable, as the performance of US is possibly influenced by body composition. Another limitation is that the sample size of our study allowed to detect a difference of $\geq 0.15$ in the AUROC curves $(\alpha=0.05, \beta=0.80)$. Therefore, statistical differences below 0.15 could be missed. However, we deem these differences to be not clinically relevant.

In conclusion, this study shows that none of the currently used screening tools for NAFLD is superior in children with obesity. ALT and US both have only moderate diagnostic accuracy for detecting hepatic steatosis. A stepwise screening strategy combining ALT and US does not improve the diagnostic accuracy. For practical reasons of costs and availability, ALT will be the preferred primary screening tool in most settings. Physicians should be aware of the limited accuracy of these screening tools when counseling patients, and better screening tools are needed to come to effective screening strategies for this endemic disorder.

Authors's contributions B.K, J.S, O.S. and T.P. were involved in the design and conduction of the study; B.K., A.B., T.P. and O.S. collected and analyzed the data. L.D. was involved in data interpretation. B.K. and L.D. wrote the manuscript; S.F. wrote the first draft; M.B. supervised the study; All authors significantly contributed to the development and improvement of the manuscript and have approved the final content of this manuscript.

\section{Compliance with ethical standards}

Conflict of interest The authors declare that they have no conflict of interest.

Ethical approval All procedures performed in studies involving human participants were in accordance with the ethical standards of the institutional and/or national research committee and with the 1964 Helsinki declaration and its later amendments or comparable ethical standards.

Informed consent Informed consent was obtained from all individual participants included in the study.

Open Access This article is distributed under the terms of the Creative Commons Attribution 4.0 International License (http:// creativecommons.org/licenses/by/4.0/), which permits unrestricted use, distribution, and reproduction in any medium, provided you give appropriate credit to the original author(s) and the source, provide a link to the Creative Commons license, and indicate if changes were made.

\section{References}

1. Vajro P, Lenta S, Socha P, Dhawan A, McKiernan P, Baumann U, Durmaz O, Lacaille F, McLin V, Nobili V (2012) Diagnosis of nonalcoholic fatty liver disease in children and adolescents: position paper of the ESPGHAN Hepatology Committee. J Pediatr Gastroenterol Nutr 54(5):700-713

2. Wong RJ, Aguilar M, Cheung R, Perumpail RB, Harrison SA, Younossi ZM, Ahmed A (2015) Nonalcoholic steatohepatitis is the second leading etiology of liver disease among adults awaiting liver transplantation in the United States. Gastroenterology 148(3): 547-555

3. Anderson EL, Howe LD, Jones HE, Higgins JP, Lawlor DA, Fraser A (2015) The prevalence of non-alcoholic fatty liver disease in children and adolescents: a systematic review and meta-analysis. PLoS One 10(10):e0140908

4. Nobili V, Reale A, Alisi A, Morino G, Trenta I, Pisani M, Marcellini M, Raucci U (2009) Elevated serum ALT in children presenting to the emergency unit: relationship with NAFLD. Dig Liver Dis 41(10):749-752

5. Schwimmer JB, Newton KP, Awai HI, Choi LJ, Garcia MA, Ellis LL, Vanderwall K, Fontanesi J (2013) Paediatric gastroenterology evaluation of overweight and obese children referred from primary care for suspected non-alcoholic fatty liver disease. Aliment Pharmacol Ther 38(10):1267-1277

6. Hecht L, Weiss R (2014) Nonalcoholic fatty liver disease and type 2 diabetes in obese children. Curr Diab Rep 14(1):448

7. Armstrong MJ, Adams LA, Canbay A, Syn WK (2014) Extrahepatic complications of nonalcoholic fatty liver disease. Hepatology 59(3):1174-1197 
8. Koot BGP, Nobili V (2017) Screening for non-alcoholic fatty liver disease in children: do guidelines provide enough guidance? Obes Rev 18:1050-1060

9. Pacifico L, Celestre M, Anania C, Paolantonio P, Chiesa C, Laghi A (2007) MRI and ultrasound for hepatic fat quantification: relationships to clinical and metabolic characteristics of pediatric nonalcoholic fatty liver disease. Acta Paediatr 96(4):542-547

10. Pozzato C, Radaelli G, Dall Asta C, Verduci E, Villa A, Villa C, Scaglioni S, Riva E, Pontiroli AE, Cornalba G, Giovannini M (2008) MRI in identifying hepatic steatosis in obese children and relation to ultrasonography and metabolic findings. J Pediatr Gastroenterol Nutr 47(4):493-499

11. Shannon A, Alkhouri N, Carter-Kent C, Monti L, Devito R, Lopez R, Feldstein AE, Nobili V (2011) Ultrasonographic quantitative estimation of hepatic steatosis in children with NAFLD. J Pediatr Gastroenterol Nutr 53(2):190-195

12. Radetti G, Kleon W, Stuefer J, Pittschieler K (2006) Non-alcoholic fatty liver disease in obese children evaluated by magnetic resonance imaging. Acta Paediatr 95(7):833-837

13. Burgert TS, Taksali SE, Dziura J, Goodman TR, Yeckel CW, Papademetris X, Constable RT, Weiss R, Tamborlane WV, Savoye M, Seyal AA, Caprio S (2006) Alanine aminotransferase levels and fatty liver in childhood obesity: associations with insulin resistance, adiponectin, and visceral fat. J Clin Endocrinol Metab 91(11):4287-4294

14. Rehm JL, Connor EL, Wolfgram PM, Eickhoff JC, Reeder SB, Allen DB (2014) Predicting hepatic steatosis in a racially and ethnically diverse cohort of adolescent girls. J Pediatr 165(2):319325.e1

15. Molleston JP, Schwimmer JB, Yates KP, Murray KF, Cummings OW, Lavine JE, Brunt EM, Scheimann AO, Unalp-Arida A, Network NCR (2014) Histological abnormalities in children with nonalcoholic fatty liver disease and normal or mildly elevated alanine aminotransferase levels. J Pediatr 164(4):707-713 e3

16. Bohte AE, Koot BG, van der Baan-Slootweg OH, van Werven JR, Bipat S, Nederveen AJ, Jansen PL, Benninga MA, Stoker J US cannot be used to predict the presence or severity of hepatic steatosis in severely obese adolescents. Radiology 2011

17. Bossuyt PM et al (2015) STARD 2015: an updated list of essential items for reporting diagnostic accuracy studies. Bmj 351:h5527

18. Cole TJ, Bellizzi MC, Flegal KM, Dietz WH (2000) Establishing a standard definition for child overweight and obesity worldwide: international survey. Bmj 320(7244):1240-1243

19. Fredriks AM, van Buuren S, Wit JM, Verloove-Vanhorick SP (2000) Body index measurements in 1996-7 compared with 1980. Arch Dis Child 82(2):107-112

20. Matthews DR, Hosker JP, Rudenski AS, Naylor BA, Treacher DF, Turner RC (1985) Homeostasis model assessment: insulin resistance and beta-cell function from fasting plasma glucose and insulin concentrations in man. Diabetologia 28(7):412-419
21. Schwimmer JB, Dunn W, Norman GJ, Pardee PE, Middleton MS, Kerkar N, Sirlin CB (2010) SAFETY study: alanine aminotransferase cutoff values are set too high for reliable detection of pediatric chronic liver disease. Gastroenterology 138(4):1357-1364 1364.e1-2

22. Vos MB et al (2016) NASPGHAN clinical practice guideline for the diagnosis and treatment of nonalcoholic fatty liver disease in children. J Pediatr Gastroenterol Nutr

23. Saverymuttu SH, Joseph AE, Maxwell JD (1986) Ultrasound scanning in the detection of hepatic fibrosis and steatosis. Br Med J (Clin Res Ed) 292(6512):13-15

24. van Werven JR, Marsman HA, Nederveen AJ, Smits NJ, ten Kate FJ, van Gulik TM, Stoker J (2010) Assessment of hepatic steatosis in patients undergoing liver resection: comparison of US, CT, T1weighted dual-echo MR imaging, and point-resolved $1 \mathrm{H}$ MR spectroscopy. Radiology 256(1):159-168

25. Szczepaniak LS, Nurenberg P, Leonard D, Browning JD, Reingold JS, Grundy S, Hobbs HH, Dobbins RL (2005) Magnetic resonance spectroscopy to measure hepatic triglyceride content: prevalence of hepatic steatosis in the general population. Am J Physiol Endocrinol Metab 288(2):E462-E468

26. Hanley JA, McNeil BJ (1983) A method of comparing the areas under receiver operating characteristic curves derived from the same cases. Radiology 148(3):839-843

27. Alberg AJ, Park JW, Hager BW, Brock MV, Diener-West M (2004) The use of "overall accuracy" to evaluate the validity of screening or diagnostic tests. J Gen Intern Med 19(5 Pt 1):460-465

28. Maguire JL, Kulik DM, Laupacis A, Kuppermann N, Uleryk EM, Parkin PC (2011) Clinical prediction rules for children: a systematic review. Pediatrics 128(3):e666-e677

29. Mofrad P, Contos MJ, Haque M, Sargeant C, Fisher RA, Luketic VA, Sterling RK, Shiffman ML, Stravitz RT, Sanyal AJ (2003) Clinical and histologic spectrum of nonalcoholic fatty liver disease associated with normal ALT values. Hepatology 37(6):1286-1292

30. Machado MV, Cortez-Pinto H (2013) Non-invasive diagnosis of non-alcoholic fatty liver disease. A critical appraisal. J Hepatol 58(5):1007-1019

31. Mathiesen UL, Franzen LE, Aselius H, Resjo M, Jacobsson L, Foberg U, Fryden A, Bodemar G (2002) Increased liver echogenicity at ultrasound examination reflects degree of steatosis but not of fibrosis in asymptomatic patients with mild/moderate abnormalities of liver transaminases. Dig Liver Dis 34(7):516-522

32. Monteiro JM, Monteiro GM, Caroli-Bottino A, Pannain VL (2014) Nonalcoholic fatty liver disease: different classifications concordance and relationship between degrees of morphological features and spectrum of the disease. Anal Cell Pathol (Amst) 2014:526979

Publisher's note Springer Nature remains neutral with regard to jurisdictional claims in published maps and institutional affiliations. 